\title{
HYPERSPECTRAL IMAGES CLASSIFICATION BY FUSING EXTINCTION PROFILES FEATURE
}

\author{
Nanjun He ${ }^{1}$, Leyuan Fang ${ }^{1}$, Shutao Li ${ }^{1}$, Pedram Ghamisi ${ }^{2}$, Jon Atli Benediktsson ${ }^{3}$ \\ ${ }^{1}$ College of Electrical and Information Engineering, Hunan University, Changsha, China \\ ${ }^{2}$ Remote Sensing Technology Institute (IMF), German Aerospace Center (DLR) and Signal \\ Processing in Earth Observation, Munich 80333, Germany \\ ${ }^{3}$ Faculty of Electrical and Computer Engineering, University of Iceland, Reykjavik, Iceland
}

\begin{abstract}
Extinction profile (EP) is an effective feature extraction method which can well preserve the geometrical characteristics of a hyperspectral image (HSI) and by extracting the EP from first three independent components (ICs) of an HSI, three correlated and complementary groups of EP features can be constructed. In this paper, an EPs fusion (EPs-F) strategy is proposed for HSI classification by exploring spatial-spectral information within and among three EP features. In general, the EPs-F method includes two stages. In the first stage, within each EP feature, a superpixel-based composite kernel strategy is proposed to adaptively fuse the spatial information of EP and the spectral feature of HSI. Then, the obtained adaptive composite kernel is used to create a classification map for each EP. In the second stage, decision fusion is further applied on different classification maps to create the final classification result. Experiments on two real HSIs verify the effectiveness of the proposed EPs-F algorithm.
\end{abstract}

Index Terms - Classification, hyperspectral image (HSI), extinction profiles (EPs), composite kernel, decision fusion.

\section{INTRODUCTION}

In recent years, a number of classifiers have been developed for the hyperspectral image (HSI) classification. Among these methods, the support vector machines (SVMs) [1] have shown remarkable performance. However, the traditional SVMs classify image without considering contextual information, i.e., inter-pixel dependency. Therefore, the classification map obtained by the SVMs may contain much pepper and salt noise. To enhance classification performance, in [2-4], the composite kernel-based SVMs were utilized to combine both the spectral and spatial information for classification.

On the other hand, various feature extraction-based classification methods have been proposed. Among these methods, morphological profile (MP)-based methods have attracted much attention, since MPs can effectively extract spatial and contextual information with low computational cost [5]. Furthermore, an extended MP (EMP) was developed to extract spatial information of HSI [6]. However, although the MP-based methods have shown an excellent performance on HSI classification, they adopt shape fixed structural element (SE) and the extracted information highly depends on the size of the object. In addition, MPs usually fail to represent information related to the gray-level characteristics of the regions [5]. To address the above-mentioned issues, extinction profiles (EPs) were introduced for HSI classification based on extinction filters (EFs) in [5]. Moreover, in [7], the concept of EPs has been generalized to extract spatial and contextual information from HSI, which can simultaneously preserve the geometrical characteristics of the input image, while discarding unimportant details. Moreover, to apply the EP to HSI, the independent component analysis (ICA) is used to extract a few informative features (e.g., three ICs) from the whole HSI and three main ICs of HSI can be preserved as base images to produce EPs which are 3 dimensional (3d) feature [7]. In this way, three complementary and correlated EP can be formed. The whole procedure of the EP-based feature extraction in HSI is shown in Fig. 1. As can be observed, within each EP, there exists still abundant spatial information. In addition, each EP has its own distinctive characteristics which can reflect the original HSI in different aspects.

In this paper, we propose an EPs-based Fusion (EPs-F) method to exploit the information within and among the EPs for HSI classification. The EPs-F first adopts a superpixel-based composite kernel strategy to adaptively exploit the information within each EP. In general, for each EP, many shape adaptive superpixels are created by an efficient over segmentation algorithm [8]. Then, three kernels are employed to utilize the spatial-spectral information of inter and intra superpixels. Furthermore, these three kernels are adaptively combined into a composite kernel and the composite kernel is fed into an SVM to obtain the first stage classification map for each EP. The three kernels weights are adjusted automatically based on the structure complexness of the input image. Furthermore, since there are correlated and complementary information among EPs, a decision fusion method is applied on different classification maps to obtain the final classification result.

The rest of this paper is organized as follows. In Section 2, the EPs are briefly introduced. In Section 3, the proposed EPs-F model for the HSI classification is detailed. The experimental results are given in Section 4. Finally, the conclusions are summarized in Section 5.

\section{EP BASED FEATURE EXTRACTION}




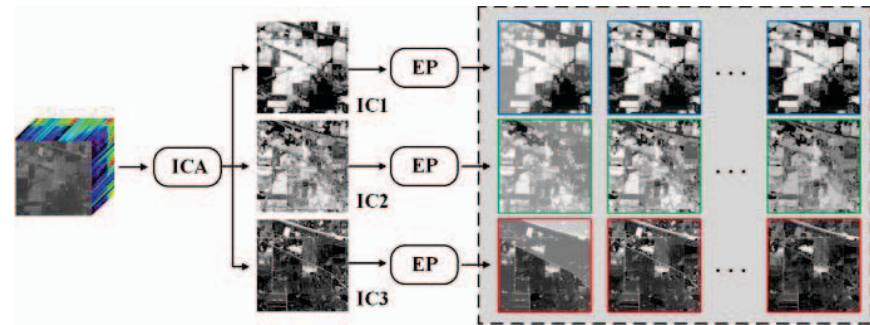

Fig. 1. The procedure to extract extinction profiles (EPs) from three independent components (ICs) of HSI.

In order to extract detailed information from an input HSI, a sequence of EF filters are taken into consideration [5]. By this way, the EPs can be produced. An EP is composed of a sequence of thinning and thickening transformations defined with a sequence of progressively stricter threshold values. An EP for the input gray-scale image, i.e., $f$, can be defined as follows:

$$
\operatorname{EP}(f)=\left\{\begin{array}{c}
\underbrace{\phi_{\lambda_{\lambda_{L}}}(f), \phi^{P_{\lambda_{L-1}}}(f), \ldots, \phi^{P_{\lambda_{1}}}(f)}_{\text {thickening profile }}, f, \\
\ldots, \underbrace{\gamma^{P_{\lambda_{L}}}(f), \gamma^{P_{\lambda_{L}}}(f), \ldots, \gamma^{P_{\lambda_{1}}}(f)}_{\text {thinning profile }}
\end{array}\right\}
$$

with $P_{\lambda_{L}}:\left\{P_{\lambda_{1}}\right\}(i=1, \ldots, L)$, a set of $L$ ordered predicates (i.e., $P_{\lambda_{i}} \subseteq P_{\lambda_{k}}, i \leq k$ ) [5]. To extend the concept of EP from a gray scale image to the HSI, the independent component analysis is first applied on the whole dimensionality to obtain the most informative components (e.g., first three ICs). Then, for each IC, we can construct the corresponding EP. In this way, three distinctive and compensative $\mathrm{EPs}$ (i.e., $\mathrm{EP}_{1}, \mathrm{EP}_{2}$, and $\mathrm{EP}_{3}$ ) can be constructed (see example in Fig. 1).

\section{PROPOSED EPs-F METHOD}

From Fig. 1, we have the following two observations: 1) There is abundant information within each EP. 2) Different EPs have their own distinctive characteristics which can offer compensative information. Therefore, in this paper, the EPs-F method is proposed to exploit the spatial information within EP and the compensative information among EPs for HSI classification.

\section{1 Stage I: Fusion within EP}

For each 3d EPs (e.g., $\mathrm{EP}_{i}, i=1,2,3$ ), the principal component analysis (PCA) is first used to reduce $\mathrm{EP}_{i}$ spectral bands and the first three principle components are used as the base image. Moreover, the entropy rate superpixel (ERS) [8] method is applied on the base image to construct a $2 \mathrm{D}$ superpixel map, and the map is combined with the corresponding EP feature to extract the non-overlapping 3D shape-adaptive regions (i.e., $\mathrm{X}_{i}^{\text {shape }}, i=1, \ldots S_{m m} . S_{m m}$ is the number of superpixel).

For each $\mathrm{X}_{i}^{\text {shape }}$, which includes a group of neighbor spectral pixels of $\mathrm{EP}_{i} x_{i}^{k}, k=1, \ldots, K$ where $K$ is number of spectral pixels, two types of spatial features are extracted:

1) inter shape-adaptive spatial feature, For the inter shape-adaptive spatial feature, we first compute the mean of spectral pixels $x_{i}^{k}, k=1, \ldots, K$ which is denoted by $\bar{x}_{i}$, and then $\bar{x}_{i}$ is assigned to every spectral pixels in $\mathrm{X}_{i}^{\text {shape }}$ to construct a mean feature $\mathrm{EP}_{i}^{\text {mean }}$.

2) intra shape-adaptive spatial feature. For the intra shape-adaptive spatial feature, a weight average strategy is applied on the neighbor superpixels (i.e., $\left.\mathrm{X}_{i, j}^{\text {shape }}, j=1, \ldots, J\right)$ of $\mathrm{X}_{i}^{\text {shape }} . J$ is the number of neighbor superpixels. In detail, the weighted average operation is applied on the mean pixels $x_{i, j}^{\text {mean }}, j=1, \ldots, J$, which are the average of $\mathrm{X}_{i, j}^{\text {shape }}$, as follows:

$x_{i}^{\text {wa }}=\sum_{j=1}^{J} w_{i, j} \times x_{i, j}^{\text {mean }}, w_{i, j}=\frac{\exp \left(-\left\|x_{i}^{\text {mean }}-x_{i, j}^{\text {mean }}\right\|_{2}^{h} / h\right)}{\text { Sum }}$ where Sum is defined as $\sum_{j=1}^{J} \exp \left(-\left\|x_{i}^{\text {mean }}-x_{i, j}^{\text {mean }}\right\|_{2}^{h} / h\right)$, and $h$ is a predefined scalar. Finally, the $x_{i}^{w a}$ is assigned to all pixels in $\mathrm{X}_{i}^{\text {shape }}$. In this way, the weight average feature image $E P^{\text {weight }}$ can be constructed.

After extracting two types of spatial features ( $\mathrm{EP}_{i}^{\text {mean }}$ and $\mathrm{EP}_{i}^{\text {weight }}$ ), the composite kernel strategy which integrates the two types of spatial features and spectral pixel is used to develop SVM classifier for creating the classification map. Specifically, for $\mathrm{EP}_{i}^{\text {mean }}, \mathrm{EP}_{i}^{\text {weight }}$ and original HSI, i.e., $I$, three kernels are created for the selected training samples which are denoted by, $K_{\text {mean }}^{\text {train }}, K_{\text {weightEP }}^{\text {train }}$, and $K_{I}^{\text {train }}$, respectively. Moreover, an adaptive strategy is proposed in which all weights are automatically adjusted based on the structure complexness (e.g., the edge information) of the input image to combine the three kernels together, as follows:

$$
K_{C K}=\mu_{1} K_{\text {meanEP }}^{\text {train }}+\mu_{2} K_{\text {weightEP }}^{\text {train }}+\mu_{3} K_{I}^{\text {train }}
$$

where $\mu_{1}, \mu_{2}$, and $\mu_{3}$ are determined by the structural complexness (detected by canny operator) of $\mathrm{EP}_{i}^{\text {mean }}$, $\mathrm{EP}_{i}^{\text {weight }}$ and $I$ respectively, while $\mu_{1}+\mu_{2}+\mu_{3}=1$. Furthermore, the obtained composite kernel $K_{C K}$ is then fed into a SVM for creating the first stage classification map. It worth noting that the Radial basis function (RBF) kernel is used in our method. The whole procedure of fusion stage I in $\mathrm{EP}_{1}$ is summarized in Fig. 2 and the aforementioned operations are applied in each EP. In this way, three compensatory and correlated classification maps can be obtained which are denoted by $\operatorname{map}_{E P_{1}}, \operatorname{map}_{E P_{2}}$ and $m a p_{E P_{3}}$ (see the example in Fig. 3). 


\subsection{Stage II: Fusion among EPs}

Since each EP has its own distinctive characteristics which can reflect the original HSI in different aspects, the classification maps ( $\operatorname{map}_{E P_{1}}, \operatorname{map}_{E P_{2}}$ and $\left.\operatorname{map}_{E P_{3}}\right)$ obtained from different EPs have compensatory and correlated information. For instance, from the red circle of Fig. 3, we can observe that the classification $\operatorname{map}_{E P_{l}}$ delivers the best classification performance in this area, while $\operatorname{map}_{E P_{2}}$ and $\operatorname{map}_{E P_{3}}$ have some wrong labels at different position. The same situation can also be observed at other zooms (e.g., the green circle and the yellow circle). Thus, in this stage, the decision fusion (e.g., majority voting) is further utilized to fuse correlated information of three obtained classification maps to create the final classification result.

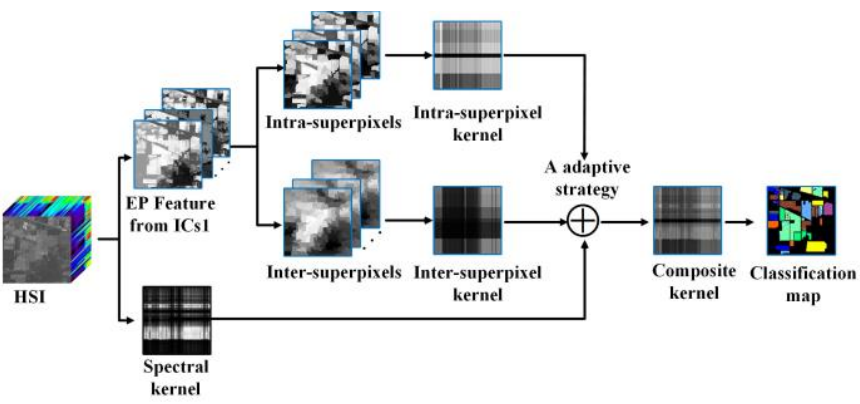

Fig. 2. The floatchart of fusion stage $I$ in $E_{1}$

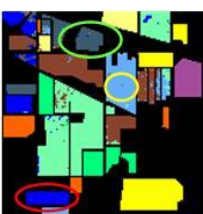

(a)

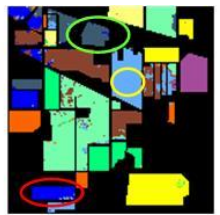

(b)

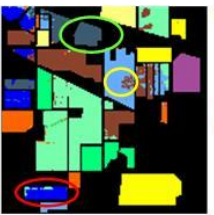

(c)

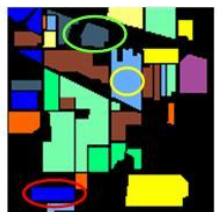

(d)
Fig. 3. (a), (b) and (c) are the classification maps (i.e., $\operatorname{map}_{E P_{l}}$, $\operatorname{map}_{E P_{2}}$ and $\operatorname{map}_{E P_{3}}$ ) obtained in the first stage, respectively. (d) is the reference map.

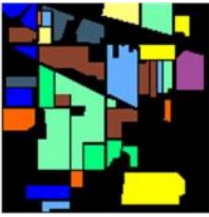

(a)

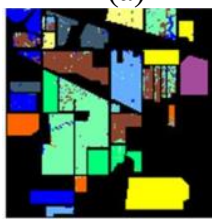

(e)

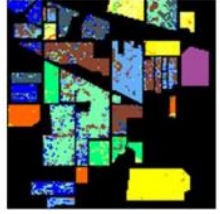

(b)

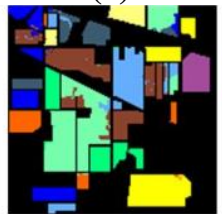

(f)

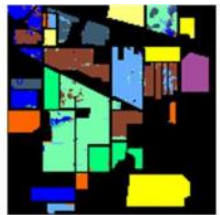

(c)

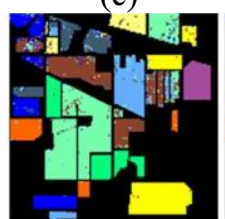

(g)

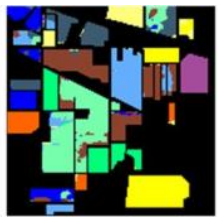

(d)

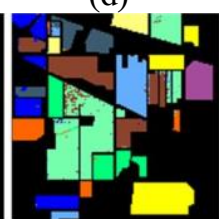

(h)
Fig. 4. Reference map and classification results for the Indian
Pines image. (a) Reference, (b) SVM $(\mathrm{OA}=74.0 \%)$, (c) EMP $(\mathrm{OA}=90.8 \%), \quad$ (d) $\mathrm{EPF} \quad(\mathrm{OA}=88.5 \%), \quad$ (e) GCK-MLR $(\mathrm{OA}=91.2 \%), \quad(\mathrm{f}) \quad \mathrm{SC}-\mathrm{MK} \quad(\mathrm{OA}=94.8 \%), \quad(\mathrm{g}) \quad \mathrm{MNFL}$ $(\mathrm{OA}=91.2 \%)$ and $(\mathrm{h})$ the proposed EPs-F $(\mathrm{OA}=96.0 \%)$.

\section{EXPERIMENTAL RESULTS}

In this section, the effectiveness of the proposed EPs-F method is tested on two hyperspectral datasets, i.e., Airborne Visible/Infrared Imaging Spectrometer (AVIRIS) Indian Pines image, Reflective Optics System Imaging Spectrometer (ROSIS-03) University of Pavia image. The classification results of the proposed EPs-F method on these two images are visually and quantitatively compared with several well-known HSI classifiers, i.e., SVM [1], EMP [6], EPF [9], GCK-MLR [2], SC-MK [4], and MNFL [10]. To objectively evaluate the classification results, three metrics of $\mathrm{OA}$, average accuracy (AA), and Kappa coefficient are used.

\subsection{Parameters setting}

For the EPs-F, the parameter $h$ in (3) is set to 500 and the parameter of SVM classifier in our method is selected by 5-fold cross-validation. The parameters of the SVM method are determined by the 10 -fold cross-validation technique. The parameters of EMP, EPF, GCK-MLR, SC-MK and MNFL are set as the defaulted values in [1], [6], [9], [2], [4] and [10], respectively

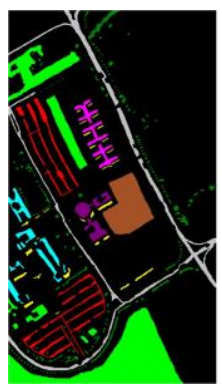

(a)

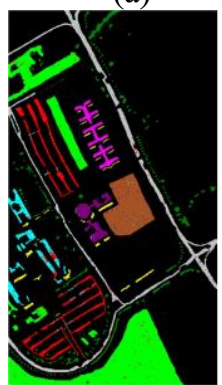

(e)

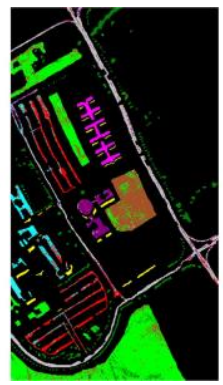

(b)

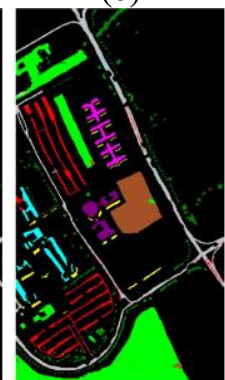

(f)

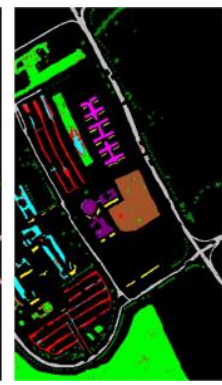

(c)

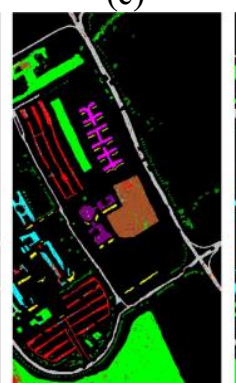

(g)

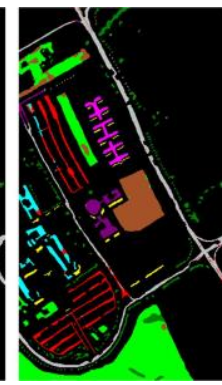

(d)

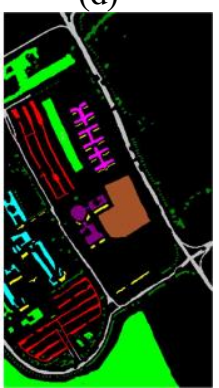

(h)
Fig. 5. Reference map and classification results for the University of Pavia image. (a) Reference, (b) SVM $(\mathrm{OA}=86.0 \%)$, (c) EMP $(\mathrm{OA}=93.9 \%)$, (d) EPF $(\mathrm{OA}=92.9 \%)$ (e) GCK-MLR $(\mathrm{OA}=96.5 \%)$, (f) SC-MK $(\mathrm{OA}=96.9 \%)$, (g) MNFL $(\mathrm{OA}=94.2 \%)$ and $(\mathrm{h})$ the proposed EPs-F $(\mathrm{OA}=99.1 \%)$. 
Table 1: Classification accuracies (in percentage) for different methods as an average (standard deviation) after ten repeated experiments. 50 training samples are randomly selected for each class.

\begin{tabular}{|c|c|c|c|c|c|c|c|}
\hline \multicolumn{8}{|c|}{ Indian Pines } \\
\hline Method & SVM [1] & EMP [6] & EPF [9] & "GCK-MLR [2] & SC-MK [4] & MNFL[10] & EPs-F \\
\hline $\mathrm{OA}$ & $72.73(0.85)$ & $89.43(1.67)$ & $88.31(1.80)$ & $91.71(0.72)$ & $95.06(0.67)$ & $90.86(0.80)$ & $95.85(0.46)$ \\
\hline AA & $68.72(0.93)$ & $87.76(1.90)$ & $86.51(2.01)$ & $94.02(0.40)$ & $96.50(0.40)$ & $91.85(0.78)$ & $97.09(0.24)$ \\
\hline Kappa & $73.50(0.60)$ & $90.00(1.61)$ & $88.20(1.52)$ & $90.40(0.22)$ & $94.30(0.83)$ & $89.40(0.92)$ & $95.20(0.54)$ \\
\hline \multicolumn{8}{|c|}{ University of Pavia } \\
\hline Method & SVM [1] & EMP [6] & EPF [9] & GCK-MLR [2] & SC-MK [4] & MNFL[10] & EPs-F \\
\hline $\mathrm{OA}$ & $84.06(1.09)$ & $94.57(0.77)$ & $93.57(2.39)$ & $96.40(\mathbf{0 . 3 6})$ & $96.28(1.48)$ & $94.95(0.72)$ & $\mathbf{9 8 . 6 7}(0.67)$ \\
\hline AA & $79.35(1.26)$ & $92.85(1.01)$ & $91.60(3.03)$ & $96.34(0.31)$ & $97.51(0.70)$ & $94.91(0.36)$ & $99.18(0.18)$ \\
\hline Kappa & $82.01(0.92)$ & $93.21(1.02)$ & $92.80(2.55)$ & $95.20(\mathbf{0 . 5 3})$ & $0.951(1.92)$ & $0.934(0.92)$ & $\mathbf{9 8 . 2 0}(0.91)$ \\
\hline
\end{tabular}

\subsection{Classification results comparison}

The first experiment is conducted on the Indian Pines image which has 220 bands of size $145 \times 145$ (20 water absorption bands were removed before experiments). The ten major classes are used in our experiment. For each class, only 50 training samples are randomly selected and the classification maps and OA are shown in Fig. 4. As can observed, the proposed EPs-F method has the highest OA (96.0\%) with less salt and pepper noise. The corresponding quantitative classification results (the average and standard variance of 10 runs) compared with other methods are presented in Table 1. As shown in Table 1, the proposed method delivers the best performance in terms of the OA, AA, and Kappa metrics.

The second experiment is performed on the University of Pavia image with the size of $610 \times 340$ pixels. It comprises 103 spectral channels and nine ground truth classes. For each class, we also selected 50 training samples. The classification maps obtained by different methods are shown in Fig. 5. From Fig. 5, we can observe that the classification result of the proposed EPs-F method produces the best visual performance with the highest $\mathrm{OA}$ (99.1\%). The corresponding quantitative classification results (the average and standard variance of 10 runs) compared with other methods are also tabulated in Table 1. From Table 1, we can also observe that, the proposed method outperforms the other investigated methods in terms of OA, AA and Kappa metrics.

\section{CONCLUSIONS}

This paper presents a novel feature fusion method named EPs-F to utilize the information of within and among EPs features for HSI classification. Within each EP, three composite kernels are employed to fuse the spatial information of the EP and spectral features of the HSI. Then, an adaptive kernel strategy is used to combine the three kernels and the fused kernel is fed into an SVM classifier to create the classification maps. In addition, among EPs, since the classification maps from different EP should contain correlated and complementary information, the decision fusion is further adopted to fuse the obtained classification maps for generating the final classification result. Our experiment on two real HSI datasets demonstrate the superiority of the proposed EPs-F method over several well-known classifiers in terms of visual quality and quantitative metrics.

\section{ACKNOWLEGEMENTS}

This paper is supported by the National Natural Science Fund of China for Distinguished Young Scholars (No. 61325007), the National Natural Science Fund of China for International Cooperation and Exchanges (No. 61520106001), the National Natural Science Foundation of China (No. 61601179), and the Science and Technology Plan Projects Fund of Hunan Province (No. 2015WK3001).

\section{REFERENCES}

[1]F. Melgani and L. Bruzzone, "Classification of hyperspectral remote sensing images with support vector machines," IEEE Trans. Geosci. Remote Sens., vol. 48, pp. 1778-1790, Aug. 2004.

[2]P. M. J. Li, A. Plaza, J. Bioucas-Dias, and J. A. Benediktsson, "Generalized Composite Kernel Framework for Hyperspectral Image Classification," IEEE Trans. Geosci. Remote Sens., vol. 51, Sep. 2013.

[3]G. Camps-Valls, L. Gomez-Chova, J. V.-F. J. Muñoz-Marí, and J. Calpe-Maravilla, "Composite kernels for hyperspectral image classification," IEEE Geosci. Remote Sens. Lett.,, vol. 3, pp. 93-97, Jan. 2006.

[4]L. Fang, S. Li, Wuhui Duan, J. Ren, and J. A. Benediktsson, "Classification of Hyperspectral Images by Exploiting Spectral-Spatial Information of Superpixel via Multiple Kernels," IEEE Trans. Geosci. Remote Sens., vol. 53, pp. 6663-6673, Dec. 2015

[5]Pedram Ghamisi, R. Souza, J. A. Benediktsson, X. X. Zhu, Letícia Rittner, and R. A. Lotufo, "Extinction Profiles for the Classification of Remote Sensing Data," IEEE Trans. Geosci. Remote Sens., vol. 54, Oct. 2016.

[6] J. A. Benediktsson, J. A. Palmason, and J. R. Sveinsson, "Classification of hyperspectral data from urban areas based on extended morphological profiles," IEEE Trans. Geosci. Remote Sens., vol. 43, Mar. 2005.

[7]P. Ghamisi, R. Souza, J. A. Benediktsson, L. Rittner, R. Lotufo, and X. X. Zhu, "Hyperspectral data classification using extended extinction profiles," IEEE Geos. Remote Sens. Let., vol. 13, pp. 1641-1645, 2016.

[8]M.-Y. Liu, O. Tuzel, S. Ramalingam, and R. Chellappa, "Entropy rate superpixel segmentation," in Proc. IEEE Conf. Comput. Vis. Pattern Recog., 2011.

[9]X. Kang, S. Li, and J. A. Benediktsson, "Spectral-Spatial Hyperspectral Image Classification With Edge-Preserving Filtering," IEEE Trans. Geosci. Remote Sens., vol. 52, pp. 2666-2677, May 2014.

[10]J. Li, X. Huang, Paolo Gamba, J. M. Bioucas-Dias, L. Zhang, J. A. Benediktsson, et al., "Multiple Feature Learning for Hyperspectral Image Classification," IEEE Trans. Geosci. Remote Sens., vol. 53, pp. 1592-1606, Mar. 2015. 